\title{
Hematopoietic and Lymphoid Cell
}

National Cancer Institute

\section{Source}

National Cancer Institute. Hematopoietic and Lymphoid Cell. NCI Thesaurus. Code C32725.

One of the cells of the blood or bone marrow, a leukocyte or erythrocyte. 